\title{
Tactile retention: Reading with the skin
}

\author{
MORTON A. HELLER \\ Winston-Salem State University, Winston-Salem, North Carolina 27102
}

\begin{abstract}
In four experiments, subjects were required to identify sequences of random digits, letters, or words drawn on the skin. The passive tactile digit span was determined to be three items when the digits were drawn with an interstimulus interval of $1 \mathrm{sec}$. Identification was significantly better for words than for random letter sequences. Slower rates of presentation produced better tactile retention. The deficiencies in passive retention were ascribed to two processes, the generation of aftersensations and a difficulty in the generation of a unitary percept. Perceptual activity may normally act to inhibit aftersensations and facilitate the construction of a unitary percept The results supported the notion that the tactile span of immediate memory is labile and may be limited by passivity.
\end{abstract}

A person typically depends upon a moving hand to explore the world. This is expecially the case when an observer attempts to obtain spatial information about an object in the environment. Katz (see Krueger, 1970) viewed the hand as an exploratory organ, and argued that passivity produces a subjective experience and a "partial anesthesia." More recently, Gibson (1962) has characterized touch as active when information is obtained from the world as a result of self-generated sensory feedback. Touch is passive when things in the environment impose themselves upon a motionless individual. Passive perception has been extensively studied in the laboratory and it has been assumed that the principles derived from such studies are generalizable to active cognitive situations. It is the purpose of this paper to evaluate the adequacy of passive retention of tactile information. It is possible that different retention mechanisms are operative in passive touch. If so, caution should be used in the interpretation of data derived from the study of passive perceptual situations.

The distinction between active and passive touch has been controversial. Some researchers have argued that passive touch is inadequate and that active perceptual exploration is necessary for the generation of veridical information about the world (Bach-y-Rita, 1972; Gibson, 1962, 1966; Heller, 1974; Heller \& Leventhal, 1975; Neisser, 1976). Heller (1974) found that active touch produced better form recognition than drawing on the skin or the static pressing of an outline form on the skin, both forms of passive touch. Although, Schwartz, Perey, and Azulay (1975) found that passive touch was no less accurate than active discrimination of form, their subjects required less time to make shape discriminations using active touch. Furthermore, Magee and Kennedy found that meaningful haptic forms might be better recognized when touch was passive (Magee \& Kennedy, 1976, as reported by Kennedy, 1978).

The drawing of numerals on the skin of the palm, or graphesthesia, is a variant of passive touch that is poorly understood (Critchley, 1953). Clinical neurologists have used the ease of recognition of such numerals as an index of normal parietal lobe, despite a lack of normative data. It has generally been assumed that it is easy to recognize numerals or letters when they are drawn on the skin (Gordon, 1978). Lashley (1951) expressed a similar view when he states: "The shape of an object can scarcely be detected with simultaneous pressure, but the same shape can readily be distinguished by touch when traced on the skin with a moving point or when explored by tactile scanning." Active touch has been shown to be better than the static deformation of the skin (Gibson, 1962; Schwartz et al., 1975). However, the adequacy of graphesthesia has not been quantified.

A number of researchers have studied passive tactile memory. Gilson and Baddeley (1969) found that retention of the locus of a tactile stimulus was affected by rehearsal and retention interval. Sullivan and Turvey (1972) found that retention decrements asymptoted after only $5 \mathrm{sec}$. More recently, Heller and Leventhal (1975) found that the retention of form information after forms were drawn on the skin decayed with time, but retention after $15 \mathrm{sec}$ was no worse than after $5 \mathrm{sec}$. They concluded that the memory trace for passive touch persists in the form of an icon that decays within 2 sec.

Watkins and Watkins (1974) reported evidence for a representational tactile memory in their study of a tactile suffix effect. Sequences of punctate stimuli were applied to the phalanges of the fingers of subjects, who were then required to recall, by a number code, the sequence of fingers that was tapped. After a sequence of eight stimulations, an auditory or tactile stimulation was provided which served as a cue for recall. It was found that the tactile recall cue, or suffix, interfered with retention of the last items on the list. They suggested that tactile information exists in the form of a sensory image that persists for 
several seconds. This was susggested by subject reports that they could still "feel" the last few stimuli at the time of recall. Gmuer and Manning (Note 1) found tactile and visual suffix effects when sequences of six letters were drawn on the skin or a visual display.

It has been suggested that passivity can have an adverse effect upon retention. Neisser (1976) has argued that when a stimulus is not anticipated or fails to lead to perceptual activity, the trace formed by that stimulus will be transient. Bach-y-Rita (1972) and White, Saunders, Scadden, Bach-y-Rita, and Collins (1970) have hypothesized that perceptual passivity may lead to transient memory traces. White et al. claimed that perceptual activity is necessary for the externalization of objectivization of sensation. People normally ignore or inhibit sensations that are interpreted as coming from within their bodies. Therefore, percepts may fade, since they are localized within the observer rather than in the outside world (Bach-y-Rita, 1972). Heller and Leventhal (1975) proposed that perceptual passivity may impede storage of a memory trace by making it very difficult for the observer to construct a unitary percept. They reasoned that activity aids in the formation of such a percept. Thus, when one actively feels an object with two fingers, one object is noticed. Such unity is not a characteristic of passive touch. For example, James (1890) noted that if an object is placed between two fingers, two contacts are felt, while one object is experienced if the two fingers are squeezed together. This view of the significance of a unitary percept is consistent with the Gestalt notion that the stability of a trace is a function of its organization (Koffka, 1935). Asch (1969) has reported evidence that recall for unitary patterns is better than recall for nonunitary patterns. Perceptual passivity may increase the load on memory because of the generation of poorly organized traces. A unitary percept can be viewed as equivalent to a Gestalt, or good form. The formation of unitary percepts may constitute a basic form of chunking.

The first experiment herein reported is an attempt to evaluate the adequacy of passive tactile retention by a modification of the procedure normally used to determine the span of immediate memory. According to Boring (1933), the span of apprehension has traditionally been taken to be six. In an important paper, Miller (1956) claimed that channel capacity was approximately seven. Crannell and Parrish (1957) found that the immediate memory span for digits was greater than that for letters or words. No effect was found when the letter or word lists were limited to a pool of nine different items. The Crannell and Parrish (1957) findings were consistent with Miller's assumptions about a fixed limit to the capacity of immediate memory. Millar (1978) reported that using active touch and memory probe technique, children could remember order information on Braille letter sequences that were six items long. It was hypothesized that the span of apprehension would be much shorter than this for lists of digits that were passively drawn on the skin of observers.

\section{EXPERIMENT 1}

The purpose of the first experiment was to determine the cutaneous digit span. Random sequences of digits were drawn on the palms of subjects. The subjects were required to recall the digits in the order in which they were presented. The procedure was basically the same as that for the determination of an auditory or visual span. Independent groups of subjects were required to identify a single digit or varying lengths of sequences of digits.

\section{Method}

Subjects. The subjects were 50 undergraduate student volunteers. The students were randomly assigned to the five sequence-length conditions with the restriction that equal numbers of males and females appear in each group.

Stimuli and Apparatus. Numerals were drawn freehand on the palms of subjects. These numerals were approximately $3.75 \mathrm{~cm}$ in diameter. They were drawn with the aid of a Lucite stylus with a rounded tip that was $.16 \mathrm{~cm}$ in diameter. All digits were drawn successively at the same location on the center of the palm. An attempt was made to draw all the numerals at a constant rate with an interstimulus interval of $1 \mathrm{sec}$ for digit sequences of 2 or longer. The digits were drawn at a rapid rate that was designed to approximate the rate that would obtain if one were actually writing large numbers with ink. Each subject was individually tested and seated at a table behind a Masonite screen. The subject was required to place his/her hand through an opening at the base of the screen that was covered by an opaque black cloth. The experimenter sat across from the subject and drew the numerals in an orientation that was upright for the experimenter. Each subject was exposed to 20 trials that consisted of the 10 individual digits presented twice in random order for the 1-digit lists. A random digits table was also used to construct 2-, 3-, 4-, and 5-digit sequence lists. The digits consisted of all the numerals from zero through nine.

Procedure. The experimental variable consisted of sequence length. Each subject was exposed to either a list consisting of 20 individual digits or one sequence length. The subject was instructed to place his/her preferred hand through the aperture in a screen with the palm facing up. Each subject was told that an individual digit or a sequence of digits would be drawn, whichever was appropriate. If a sequence was drawn, the subject was told the length of the sequence. Subjects were told to identify the number or number sequence when given a verbal ready signal immediately after the individual digit or sequence was drawn. When given the ready signal, the subjects were instructed to repeat the sequence in exactly the same order as it was drawn. Instructions to guess were given to the subjects when they were unsure about a response. No feedback was provided until all 20 trials were completed. Each trial consisted of the presentation of a number and a verbal identification by the observer.

\section{Results and Discussion}

The immediate memory span was strikingly poor for drawing on the skin. Table 1 shows the mean percentage of correct responses for each sequence length. If the digit span is taken as the $50 \%$ point as Boring (1933) and Woodworth and Schlosberg (1954) suggest, 
Table 1

Mean Number Correct, Percent Correct, and Standard Deviation for Each Sequence Length

\begin{tabular}{cccc}
$\begin{array}{c}\text { Sequence } \\
\text { Length }\end{array}$ & $\begin{array}{c}\text { Mean } \\
\text { Percent } \\
\text { Correct }\end{array}$ & $\begin{array}{c}\text { Mean } \\
\text { Number } \\
\text { Correct }\end{array}$ & $\begin{array}{c}\text { Standard } \\
\text { Deviation }\end{array}$ \\
\hline 1 & 82.0 & 16.4 & 2.0 \\
2 & 71.0 & 14.2 & 4.5 \\
3 & 51.5 & 10.3 & 5.0 \\
4 & 39.5 & 7.9 & 4.9 \\
5 & 35.0 & 7.0 & 3.5 \\
\hline
\end{tabular}

the passive cutaneous digit span is merely three items. An analysis of variance showed that the effect of sequence length was significant $[F(4,45)=8.62, p<$ .011 .

Channel capacity does not seem to be an immutable number. The results of the first experiment are consistent with Neisser's (1976) assertion that channel capacity is labile. In particular, the results suggest that perceptual passivity may serve to severely limit the span of apprehension.

The subjects invariably reported a tingling aftersensation resulting from drawing on the skin that persisted until another item was drawn. Many subjects reported the experience of an image or icon that was described as a literal copy of the stimulation. Either type of persistent sensory experience was variably described as wiping out either a subsequent or prior tactile image. Some subjects remarked that the numbers seemed to blend into each other such that they could not always tell when one digit ended and another began. They thought the task would have been easier with longer interstimulus intervals. It must be noted that a variety of stimuli have been known to produce aftersensations when the observer is passive (Hayes, 1912). Hayes reported that punctate and areal stimulation reliably yield aftersensations. Points moving across the skin have also been reported to yield aftersensations of variable durations.

\section{EXPERIMENT 2}

A second experiment was performed to test the generality of the results of the first study. The purpose of this experiment was to determine if organization of tactile material into familiar units would aid in the retention of letter sequences. The procedure was similar to that of the first experiment, with a few differences. Letters were drawn on the palms of some subjects, while words were drawn on the palms of others. Words constitute an obvious form of chunking, and should have significantly improved the transmission of information.

\section{Method}

Subjects. Twenty-four student volunteers participated in the study. Equal numbers of males and females were assigned ran- domly to the two experimental conditions. It should be noted that many subjects reported that as children they played at writing messages on the backs or palms of other children.

Stimuli and Apparatus. The letters used for the random-letters group consisted of the printed capital letters $A$ through $J$, in random order. The subjects were exposed to two-, four-, and six-letter sequences. Other subjects were required to identify two-, four-, and six-letter words. The two-letter words were: at, in, by, am, do, be, we, it, go, and my. The four-and six-letter words were common nouns with high recall scores taken from Christian, Bickley, Tarka, and Clayton (1978). The four-letter words were: army, hall, frog, bowl, jury, gift, corn, iron, duty, and monk. The six-letter nouns were: heaven, dollar, equity, animal, letter, injury, flower, garden, cattle, and bandit. Letters in the word condition were also written as capitals. Thus, each letter-condition subject was exposed to 10 two-letter sequences, then 10 four-letter sequences, and finally 10 six-letter sequences. Word-condition subjects were also started off with the shortest words, then exposed to four-letter words, and finally to six-letter words.

Procedure. The procedure was similar to that of the first experiment. Subjects were instructed that the letters A through J would be drawn for the letter condition and were instructed to guess if unsure. They were told when a two-letter sequence would be drawn and provided further information on sequence length when the longer sequences were used. Each letter subject was exposed to 30 trials, consisting of 10 two-, four-, and six-letter sequences. Subjects in the word condition were told that words would be drawn on their palms and were instructed to identify the word after it was written. These subjects were also provided information about word length. Each word subject had to "read" 10 two-, four-, and six-letter words, for a total of 30 trials. Word subjects were informed that the letters would always be drawn as capital letters, but were not provided with any further information about the nature of the words, that is, they were not told that the longer words were nouns. As in the first experiment, the subjects were provided with a verbal cue for recali.

\section{Results and Discussion}

The data clearly show an effect of sequence length and organization upon recall. Shorter sequences, not surprisingly, were far easier to recall. Words were easier to recall than random sequences of letters. Table 2 shows the proportion of words and letter sequences correctly identified.

The experiment had the form of a two-factor experiment, the factors being organization of stimuli (words vs. letters) and sequence length, with repeated measures on the last factor. A mixed-factor analysis of variance showed that the effect of organization was significant $[F(1,22)=8.42, p<.01]$. The effect of list length was also significant $[\mathrm{F}(2,44)=90.41, \mathrm{p}<.01]$. However, the interaction effect was not significant $[F(2,44)=1.27, \mathrm{p}>.05]$. A Tukey (hsd) test comparing the three word length means, two at a time,

Table 2

Proportion of Correct Identifications of Sequences and Words

\begin{tabular}{lccc}
\hline & \multicolumn{3}{c}{ Mean Percent Correct } \\
\cline { 2 - 4 } & \multicolumn{3}{c}{ Sequence Length in Letters } \\
& 2 & 4 & 6 \\
\hline Letters & 39.2 & 21.7 & 4.2 \\
Words & 59.2 & 33.3 & 16.7 \\
\hline
\end{tabular}


showed that mean recall for two-letter words was significantly better than that for longer words $(p<.01)$. Four-letter words were identified with greater mean accuracy than longer words $(p<.01)$. A further Tukey (hsd) test showed that two-letter sequences were better recalled than longer sequences $(p<.01)$. Four-letter random sequences were recalled with greater man accuracy than six-letter sequences $(p<.01)$.

It was surprising that the random-letter group did so poorly. Subjects in this condition showed less adequate memory than did subjects with digit sequences. To determine if this was owing to poor letter recognition, an additional group of 10 subjects was required to identify individual letters. The procedure was similar to that in the second experiment, and a limited letter list was also used. The subjects were required to identify each of the letters in this limited list immediately after its presentation, for 20 trials. These subjects were told that the letter list was limited to the letters $A$ through $\mathbf{J}$. The mean percentage of letters correctly identified was $\mathbf{8 2 \%}$. This performance was identical to that for digits. It should be noted that $47.9 \%$ of the letters were recalled correctly in the second experiment when two letters were presented serially, and only $39.2 \%$ of the sequences of two letters were recalled correctly. Poor letter recognition cannot be used to explain the inadequate memory that was obtained for serial letter recall, since letter recognition was comparable to digit recognition. It must be noted that the superiority of recall for digit sequences in the present study is consistent with similar findings for the auditory presentation of digits and letters by Crannell and Parrish (1957), who asserted that subjects may be more familiar with random digit sequences and consequently recall them better.

\section{EXPERIMENT 3}

A third experiment was performed to determine if the results of the first two experiments were specific to the inverted orientation with which symbols were drawn. It was considered possible that the low level of performance obtained for passive touch may have been a function of the orientation of the drawn forms. Random four-letter sequences were drawn on the palms of subjects. The letters were drawn upright for the experimenter, or upright for the subject, or at a right angle to the arm-axis.

\section{Method}

Subjects. The subjects were 12 undergraduate student volunteers. Equal numbers of males and females were used for the experiment.

Stimuli and Apparatus. The stimuli consisted of random fourletter sequences. The letters were limited to the capital letters, A through $\mathrm{J}$, as in the second experiment. Each subject was exposed to 30 four-letter sequences, with blocks of 10 sequences presented in each orientation. The order of presentation of the orientations was completely counterbalanced. Thus, each subject was exposed to blocks of 10 upright sequences, 10 inverted sequences, and 10 perpendicular sequences.

Procedure. The procedure was similar to that of the second experiment. The subjects were instructed about the orientations and told when the orientation of the letter sequences would be changed. They were also told that the tops of the letters would be oriented toward the root of the fingers for the upright orientation. For the perpendicular orientation, the experimenter stood to the left of the subject and drew the letters at a right angle to the arm-axis. The procedure was identical to that in the second experiment for the inverted orientation.

\section{Results and Discussion}

Orientation has no effect on the retention of letter sequences. The mean frequencies of sequences correct for the upright, perpendicular, and inverted orientations were $19.1 \%, 21.7 \%$, and $15 \%$, respectively. The effect of orientation was not significant $[F(2,22)=$ $.56, \mathrm{p}>.05]$. The obtained scores are comparable to those for four-letter sequences in the second experiment. This suggests that the low level of retention obtained for passive touch cannot be ascribed to the orientation of the stimuli.

\section{EXPERIMENT 4}

A fourth experiment was performed to determine if aftersensations might have contributed to the observed tactile forgetting. The skin has been described by some researchers as suffering from limited temporal resolution (Kirman, 1973). It was expected that slowed-down tactile presentations would permit aftersensations to dissipate and facilitate tactile reading. Words were drawn on the palms of subjects with 1-, 3-, or 5-sec intervals between letters.

\section{Method}

Subjects. There were 30 subjects in the experiment. Equal numbers of males and females were randomly assigned to the three interstimulus interval conditions.

Stimuli and Apparatus. The subjects were required to read words that were drawn on the skin. All subjects were exposed to three-, five-, and seven-letter nouns with high recall scores, taken from Christian et al. (1978). The three-letter words were: gem, boy, air, joy, fox, bar, lad, sea, cat, and keg. The five-letter words were: ghost, anger, death, brute, party, cabin, shock, judge, flesh, and horse. The seven-letter words were: college, fortune, lecture, victory, student, disease, boulder, teacher, pudding, and science. All letters were printed as capitals.

Procedure. The experimental variable was interstimulus interval. Independent groups of subjects were required to read words that were drawn with an interstimulus interval of 1,3 , or $5 \mathrm{sec}$ between the letters. The procedure was similar to that of the second experiment. Each subject was exposed to 30 trials in which they had to read 10 three-, five-, and seven-letter words. As in the second experiment, subjects were informed about word length. The subjects started with the shortest words, and then attempted to read progressively longer words. The letters were drawn in an upright orientation for the subjects, since several subjects expressed a preference for that procedure in the third experiment.

\section{Results and Discussion}

Presentation rate was found to have an effect upon the ease of identification of words drawn on the skin. 
Slower rates resulted in more accurate reading with the skin. Longer words were not more difficult for the slower presentation rates. Table 3 shows the proportion of long and short words correctly identified at each rate of presentation. Slowed-down presentations appear to be especially helpful in identification of the longest words studied.

The experiment had the form of a two-factor experiment, the factors being interstimulus interval and word length, with repeated measures on word length. Data analysis showed that the main effect of interstimulus interval was significant $[\mathrm{F}(2,27)=7.51, \mathrm{p}<$ $.01]$. The effect of word length was not significant $[\mathrm{F}(2,54)=.90, \mathrm{p}>.05]$. However, the interaction effect was significant $[F(4,54)=2.79, p<.05]$. Tests on simple main effects were performed. The simple main effect of interstimulus interval was significant for three-letter words $[F(2,27)=6.38, p<.05]$, fiveletter words $[\mathrm{F}(2,27)=4.86, \mathrm{p}<.05]$, and seven-letter words $[F(2,27)=12.01, p<.01]$. However, the simple main effect of word length was significant for the 1 -sec condition $[F(2,54)=4.29, p<.05]$. This suggests that the effect of word length depends upon presentation rate, since the simple main effect of word length was not significant for the two longer interstimulus interval conditions ( $p>.05$ for both comparisons).

Slower presentation rates aid in perceptual integration and allow subjects to retain longer sequences. Rapid presentation rates seem to generate a preperceptual store that is very limited in capacity. Faster rates may yield a poor perceptual organization, while subjects in the 5-sec condition were better able to use lexical cues. This was revealed by reports of subjects in the 5-sec condition that they could often anticipate a word before its completion, particularly if the word was long. Nonetheless, it must be remarked that word identification was not very accurate. Indeed, the obtained accuracy of $56 \%$ correct for three-letter words at the slowest rate represents mediocre performance. It is not known what effect, if any, training might have on passive tactile reading.

The obtained results support the hypothesis that aftersensations may have contributed to the poor retention found for passive touch. The results suggest that aftersensations ofion persist for at least $3 \mathrm{sec}$ since the 5 -sec rate produced so much improvement

Table 3

Mean Proportion of Words Identified

at Each Interstimulus Interval (ISI)

\begin{tabular}{cccc} 
& \multicolumn{3}{c}{ Mean Percent Correct } \\
\cline { 2 - 4 } ISI & \multicolumn{3}{c}{ Word Length in Let ters } \\
(Seconds) & 3 & 5 & 7 \\
\hline 1 & 30 & 25 & 12 \\
3 & 41 & 39 & 34 \\
5 & 56 & 60 & 67 \\
\hline
\end{tabular}

over the faster rates. This result is noteworthy in view of the Gilson and Baddeley (1969) assertion that aftersensations derived from punctate stimulation seemed to fade after $3 \mathrm{sec}$. However, introspective reports are variable and difficult to interpret, since at least one subject reported that words seemed to run into each other. Thus, aftersensations might last minutes for some individuals. In an extensive review of the early literature, Hayes (1912) revealed that some observers noticed that aftersensations could persist for up to $40 \mathrm{sec}$ and recur cyclically.

\section{GENERAL DISCUSSION}

The results of the experiments supported the prediction that passive retention would be poor. Perceptual passivity may interfere with normal retention mechanisms, and further impede rehearsal. Passive touch probably leads to a long-lasting preperceptual memory. Kirman (1973) has argued that a durable preperceptuai store is an essential prerequisite for effective tactile communication. The results of the present study suggest that tactile communication might be facilitated by less frequent presentations and a highly organized stimulus input, since rapid presentations seem to generate interference.

The results suggest that passivity leads to the generation of aftersensations and afterimages. These aftersensations interfere with further encoding of the stimulus material and may lead to the obliteration of the memory trace of prior items. Such interference may also be proactive. Furthermore, the sensory impression produced by passive drawing on the skin can be so attention demanding that attention is directed away from trace maintenance procedures. It should be noted that aftersensations are rarely, if ever, noticed when touch is active. It is suggested that motor activity is instrumental in the inhibition of aftersensations and facilitates the direction of attention. This analysis is derived from Weber's discussion of common sensibility (Weber, 1978/1846). Motor activity was viewed by Weber as instrumental in the generation of objective information about the world and an externalized percept. Passivity was believed to contribute to subjective sensations. Aftersensations, pain, and itch were among the sensory impressions that were classified as examples of common sensibility. Such common sensations were believed to detract from attention mechanisms. Therefore, passive touch can be viewed as generating a relatively subjective experience that interferes with rehearsal and attention. On this view, passive touch leads to retention loss because sensory traces are generated which are too durable and may have an affective component. This interpretation of the data is an alternative to Bach-y-Rita's (1972) suggestion that passive traces tend to fade in time and lead to forgetting. Rather, it is proposed that overly 
persistent sensory traces produce interference in a serial task.

The experimental outcome is in accord with the Heller and Leventhal (1975) assertion that passivity may lead to retention losses, since it is difficult to construct a unitary percept with passive drawing on the skin. Passive touch may generate poorly organized traces that are subject to interference when memory is loaded. This is suggested by subject reports that it was not always clear when one letter ended and another began. These reports also suggest that aftersensations can promote trace aggregation. It must be noted that the level of organization of a trace may also be a function of stimulus parameters. For example, introspection reveals that such letters as $G$ and $O$ are more likely to yield a unitary percept than letters such as H or F. Subjects seemed more likely to report that these latter configurations produced a simple tingling aftersensation, while the $G$ was more likely to yield an identifiable afterimage. Of course, these observations were not systematically obtained and require verification. Nonetheless, these introspective reports are not consistent with the assumption of a constancy between stimulation and perception. A commonly known visual analog to drawing on the skin may be the image that results when one twirls a burning ember in the dark to form a pattern. Under such circumstances, the unitary percept of a visual letter $O$ would be far easier for an observer to construct than a capital Q.

Clearly, perceptual passivity can lead to poor retention of verbally coded tactile information. Further research needs to be performed to isolate the mechanisms that cause interference in tactile retention. It is not clear which parameters control the duration, quality, or generation of afterimages and aftersensations. Since passive touch can be more accurate than active touch (see Kennedy, 1978), it is proposed that passive touch is especially handicapped when stimulus sequences are relatively unorganized and meaningless.

\section{REFERENCE NOTE}

1. Gmuer, B. A., \& Manning, S. K. Tactual and visual suffix effects. Paper presented at the meetings of the Eastern Psychological Association, Philadelphia, April 1979.

\section{REFERENCES}

Asch, S. E. A reformulation of the problem of associations. American Psychologist, 1969, 24, 92-102.

ВАсH-Y-R ITA, P. Brain mechanisms in sensory substitution. New York: Academic Press, 1972.

Boring, E. G. The physical dimensions of consciousness. New York: Century, 1933.

Christian, J., Bickley, W., Tarka, M., \& Clayton, K. Measures of free recall of 900 English nouns: Correlations with imagery, concreteness, meaningfulness, and frequency. Memory \& Cognition, 1978, 6, 379-390.

Crannele, C. W., \& Parrish, J. M. A comparison of immediate memory span for digits, letters, and words. Journal of Psychology, 1957, 44, 319-327.

Critchle Y, M. The parietal lobes. New York: Hafner, 1953.

Gibson, J. J. Observations on active touch. Psychological Review, $1962,69,477-490$.

GiBson, J. J. The senses considered as perceptual systems. Boston: Houghton Mifflin, 1966.

Gilson, E. Q., \& BadDEley, A. D. Tactile short-term memory. Quarterly Journal of Experimental Psychology, 1969, 21, 180-184.

Gordon, G. Introduction. In G. Gordon (Ed.), Active touch. Oxford: Pergamon Press, 1978.

HAYEs, M. H. S. A study of cutaneous after-sensations. Psychological Monographs, 1912, 14(Whole No. 60).

Heller, M. A. Some observations on passive touch. (Doctoral Dissertation, Yeshiva University) Ann Arbor: University Microfilms, 1974. (No. 75-9044)

Heller, M. A., \& Leventhal, G. Delay in retention of forms. Perceptual and Molor Skills, 1975, 41, 903-906.

James. W. The principles of psychology (Vol. 2). New York: Holt, 1890.

Kennedy, J. M. Haptics. In E. C. Carterette \& M. P. Friedman (Eds.), Handbook of perception (Vol. 8, Perceptual coding). New York: Academic Press, 1978.

Kirman, J. H. Tactile communication of speech: A review and an analysis. Psychological Bulletin, 1973, 80, 54-74.

Koffka, K. Principles of Gestalt psychology. New York: Harcourt, Brace and World, 1935.

Krueger, L. E. David Katz's Der Aufbau der Tastwelt (The world of touch): A synopsis. Perception \& Psychophysics, 1970, 7, 337-341.

LASHLEY, K. S. The problem of serial behavior. In L. A. Jeffress (Ed.), Cerebral mechanisms in behavior. New York: Wiley, 1951.

MilLAR, S. Short-term serial tactual recall: Effects of grouping on tactually probed recall of Braille letters and nonsense shapes by blind children. British Journal of Psychology, 1978, 69, 17-24.

Miller, G. A. The magical number seven, plus or minus two: Some limits on our capacity for processing of information. Psychological Review, 1956, 63, 81-97.

NeISSER, U. Cognition and reality. San Francisco: Freeman, 1976.

Schwartz, A. S., Perey, A. J., \& Azulay, A. Further analysis of active and passive touch in pattern discrimination. Bulletin of the Psychonomic Society, 1975, 6, 7-9.

Sullivan, E. V., \& Turvey, M. T. Short-term retention of tactile stimulation. Quarterly Journal of Experimental Psychology, 1972, 24, 253-261.

Watkins, M. J., \& Watkins, O. C. A tactile suffix effect. Memory \& Cognition, 1974, 2, 176-180.

WEBER, E. H. The sense of touch. (H. E. Ross \& D. J. Murray, trans.). New York: Academic Press, 1978. (Originally published, 1846.)

White, B. W., Saunders, F. A., Scadden, L., Bach-y-Rita, P., \& Collins, C. C. Seeing with the skin. Perception \& Psychophysics, 1970, 7, 23-27.

Woodworth, R. S., \& Schlosberg, H. Experimental psychology. New York: Holt, Rinehart, and Winston, 1954.

(Received for publication May 10, 1979; revision accepted November 26, 1979.) 